\title{
Discovery of a new supernova remnant G21.8-3.0
}

\author{
X. Y. Gao ${ }^{1,2 \star}$ P. Reich,${ }^{3}$ W. Reich ${ }^{3}$ L. G. Hou ${ }^{1,2}$ and J. L. Han, ${ }^{1,2,4}$ \\ ${ }^{1}$ National Astronomical Observatories, CAS, Jia-20 Datun Road, Chaoyang District, Beijing 100101, PR China \\ ${ }^{2}$ CAS Key Laboratory of FAST, National Astronomical Observatories, Chinese Academy of Sciences \\ ${ }^{3}$ Max-Planck-Institut für Radioastronomie, Auf dem Hügel 69, 53121 Bonn, Germany \\ ${ }^{4}$ School of Astronomy, University of Chinese Academy of Sciences, Beijing 100049, China
}

Accepted XXX. Received YYY; in original form ZZZ

\begin{abstract}
Sensitive radio continuum surveys of the Galactic plane are ideal for discovering new supernova remnants (SNRs). From the Sino-German $\lambda 6 \mathrm{~cm}$ polarisation survey of the Galactic plane, an extended shell-like structure has been found at $\ell=21.8, b=-3.0$, which has a size of about $1^{\circ}$. New observations were made with the Effelsberg $100-\mathrm{m}$ radio telescope at $\lambda 11 \mathrm{~cm}$ to estimate the source spectrum together with the Urumqi $\lambda 6 \mathrm{~cm}$ and the Effelsberg $\lambda 21 \mathrm{~cm}$ data. The spectral index of G21.8-3.0 was found to be $\alpha=-0.72 \pm 0.16$. Polarised emission was mostly detected in the eastern half of G21.8-3.0 at both $\lambda 6 \mathrm{~cm}$ and $\lambda 11 \mathrm{~cm}$. These properties, together with the $\mathrm{H} \alpha$ filament along its northern periphery and the lack of infrared emission, indicate that the emission is non-thermal as is usual in shell-type SNRs.
\end{abstract}

Key words: ISM: supernova remnants - ISM: individual objects: G21.8-3.0 - radio continuum: general - Methods: observational

\section{INTRODUCTION}

Supernova explosions process the Galactic interstellar medium (ISM) and enhance dynamics in the Galaxy by e.g. accelerating particles, enriching chemical elements, and triggering the next generation of star formation (Padmanabhan 2001). The remnants glow for more than hundreds of thousands years when interacting with the ISM. The total count of these Galactic supernova remnants (SNRs), as a basic parameter, is however still unclear. A large deficiency exists between the predicted ( $\mathrm{Li}$ et al. 1991; Tammann et al. 1994) and the observed number (Ferrand \& Safi-Harb 2012; Green 2019). To bridge this gap, extensive discoveries of new SNRs depend on deeper radio continuum surveys to unveil the faint cases, and sharper views to separate small structures, especially toward the inner Galaxy (e.g. Brogan et al. 2006). Recently, a big step was made by Anderson et al. (2017): through combining the radio continuum data from "The H I, $\mathrm{OH}$, Recombination line survey of the Milky Way" (THOR, angular resolution $\sim 20^{\prime \prime}$ ), the VLA $1.4 \mathrm{GHz}$ Galactic Plane Survey (VGPS, angular resolution $\sim 1^{\prime}$, Stil et al. 2006), and the Effelsberg $\lambda 21 \mathrm{~cm}$ survey (Reich et al. 1990, angular resolution $\sim 9^{\prime}$ ), they identified 76 new SNR candidates in the area of $17^{\circ} .5<\ell<67^{\circ} .4$ and $|b| \leqslant 1.25$. A number of new SNRs have also been identified through very careful inspections in arcmin-resolution images of very sensitive large-scale radio surveys (e.g. Foster et al. 2013; Kothes et al. 2014;

* E-mail: xygao@nao.cas.cn (XYG)
Gerbrandt et al. 2014; Kothes et al. 2017) and occasionally from deep $\mathrm{H} \alpha$ observations (e.g. Fesen \& Milisavljevic 2010; Fesen et al. 2015).

Using the data of the Sino-German $\lambda 6 \mathrm{~cm}$ polarisation survey of the Galactic plane ${ }^{1}$ (Sun et al. 2007; Gao et al. 2010; Sun et al. 2011; Xiao et al. 2011), we discovered three new SNRs: G25.1-2.3 and G178.2-4.2 by Gao et al. (2011) and G150.3+4.5 by Gao \& Han (2014). Here we report the discovery of a new shell-like SNR, G21.8-3.0. We introduce the basic data in Sect. 2, and discuss the properties of G21.8-3.0 in Sect. 3. A summary is given in Sect. 4 .

\section{BASIC DATA}

\subsection{Urumqi $\lambda 6 \mathrm{~cm}$ data}

The $\lambda 6 \mathrm{~cm}$ total-intensity (Stokes $I$ ) and linear-polarisation (Stokes $Q$ and $U$ ) data were extracted from the Sino-German $\lambda 6 \mathrm{~cm}$ polarisation survey of the Galactic plane. The data were acquired by the Urumqi $25-\mathrm{m}$ radio telescope of Xinjiang Astronomical Observatory, equipped with a singlechannel receiver constructed by the Max-Planck-Institut für Radioastronomie, Germany. The angular resolution of the survey is 9.5 . The sensitivity is about $1.0 \mathrm{mK} T_{\mathrm{B}}$ for $I$ and $0.5 \mathrm{mK} T_{\mathrm{B}}$ for $Q / U$ in the inner Galactic part of the survey.

1 Data available at http://www.mpifr-bonn.mpg.de/survey.html and http://zmtt.bao.ac.cn/6cm/surveydata.html 
Details on the $\lambda 6 \mathrm{~cm}$ receiving system, observation strategy, set-up and data reduction were described in Sun et al. (2006, 2007) and Gao et al. (2010). SNRs with surface brightness larger than $\Sigma_{1 \mathrm{GHz}} \sim 3.9 \times 10^{-23}\left[\mathrm{Wm}^{-2} \mathrm{~Hz}^{-1} \mathrm{sr}^{-1}\right](3 \sigma$ detection limit) could be detected in this survey (Gao et al. 2010).

An extended shell-like source, G21.8-3.0 (see Fig. 1), with associated polarised emission was found at $\ell=$ $21^{\circ} .8, b=-3^{\circ} .0$, which has a size of about $1^{\circ}$.

\subsection{Effelsberg $\lambda 11 \mathrm{~cm}$ data}

To reveal the properties of G21.8-3.0, new $\lambda 11 \mathrm{~cm}$ totalintensity and linear-polarisation observations were made with the Effelsberg 100-m radio telescope in October, 2018. We decided not to use the standard 8-channel $80-\mathrm{MHz}$ bandwidth backend centred at $2639.5 \mathrm{MHz}$, with which tests in December 2017 showed that strong radio interference (RFI) reduced the usable bandwidth to $40 \mathrm{MHz}$. Instead, the 2$\mathrm{GHz}$ wide SPECPOL polarimeter with 1024 channels available at the Effelsberg telescope was successfully attached to the receiver to check the RFI situation outside the standard $80-\mathrm{MHz}$ band. Although the spectrometer covered the band from $2300 \mathrm{MHz}$ to $4300 \mathrm{MHz}$, the filter in the $\lambda 11 \mathrm{~cm}$ frontend limits the accessible frequency range from $2599.5 \mathrm{MHz}$ to $2679.5 \mathrm{MHz}$. The test was successful and showed that in total four frequency bands were usable. The remaining RFI could be removed either by software or by data editing. The four bands cover in total about $185 \mathrm{MHz}$, which improves on the bandwidth available so far. The four bands were centred at $2376.25 \mathrm{MHz}, 2553.75 \mathrm{MHz}, 2658.0 \mathrm{MHz}$, and 2696.25 MHz and have a usable bandwidth of $32.5 \mathrm{MHz}$, 117.5 MHz, 8 MHz, and 27.5 MHz, respectively. The NOD2based data reduction of G21.8-3.0 followed the same routines as for the $\lambda 6 \mathrm{~cm}$ data (Gao et al. 2010). The angular resolutions of the four sections are slightly different, from 4.2 to 4.7. After data reduction, we added them after convolving all the images to 4.8 . The sensitivity of the combined data is about $5.0 \mathrm{mK} T_{\mathrm{B}}$ and $4.5 \mathrm{mK} T_{\mathrm{B}}$ for $I$ and $Q / U$, respectively. We note, however, that the final noise does not fully reflect the increase in bandwidth when compared to earlier $\lambda 11 \mathrm{~cm}$ observations. This indicates either the existence of low-level RFI in- or out-side the selected bands and requires further tests for optimisation in the future.

\subsection{Effelsberg $\lambda 21 \mathrm{~cm}$ data}

The $\lambda 21 \mathrm{~cm}$ total-intensity data of G21.8-3.0 came from the Effelsberg survey (Reich et al. 1990). Polarisation data were not available. The central observing frequency of the survey was $1408 \mathrm{MHz}$. The bandwidth was often set to $20 \mathrm{MHz}$. The beam size is 9'4, nearly identical to that of the Urumqi $\lambda 6$ $\mathrm{cm}$ survey. Typical r.m.s. of the noise was about $40 \mathrm{mK} T_{\mathrm{B}}$. The high angular resolution Canadian Galactic Plane Survey (CGPS, Landecker et al. 2010) and VGPS (Stil et al. 2006), which surveyed the Galactic plane both in radio continuum and $\mathrm{HI}$, do not cover this area.
Table 1. Flux densities of three strong point-like sources overlapping G21.8-3.0.

\begin{tabular}{cccc}
\hline \hline Source & $\begin{array}{c}S_{\lambda 21 \mathrm{~cm}} \\
(\mathrm{mJy})\end{array}$ & $\begin{array}{c}S_{\lambda 11 \mathrm{~cm}} \\
(\mathrm{mJy})\end{array}$ & $\begin{array}{c}S_{\lambda 6 \mathrm{~cm}} \\
(\mathrm{mJy})\end{array}$ \\
\hline G22.08-3.19 & 37.7 & $35.5^{*}$ & $33.3^{*}$ \\
$\mathrm{G} 21.93-2.72$ & 115.6 & 77.7 & 64 \\
$\mathrm{G} 21.66-2.79$ & 102.0 & 83.6 & $67.9^{*}$ \\
\hline
\end{tabular}

Notes: values with ${ }^{*}$ are extrapolated.

\subsection{Other data}

Besides the radio continuum data, additional data were also used to study the properties of G21.8-3.0. The optical H $\alpha$ data were taken from the Southern $\mathrm{H} \alpha$ Sky Survey Atlas (hereafter SHASSA, Gaustad et al. 2001). Two sets of infrared data around G21.8-3.0 were adopted for comparison with the radio continuum emission. The $60 \mu \mathrm{m}$ data were from the Improved Reprocessing of the Infrared Astronomical Satellite Survey (IRIS, Miville-Deschênes \& Lagache 2005). The $12 \mu \mathrm{m}$ and $22 \mu \mathrm{m}$ WISE data (Wright et al. 2010) and the WISE H II region catalogue ${ }^{2}$ (Anderson et al. 2014) were also consulted. In addition, the X-ray data from ROSAT all-sky survey (Voges et al. 1999) and Chandra Observatory $^{3}, \gamma$-ray sources identified in the HESS and HAWC (Jardin-Blicq et al. 2019) have also been checked for a highenergy counterpart associated with G21.8-3.0. H I data from the Parkes Galactic all-sky survey (McClure-Griffiths et al. 2009; Kalberla et al. 2010) and the ${ }^{12} \mathrm{CO}(J=1-0)$ data from Dame et al. (2001) were checked for a possible coincident structure to estimate the distance of G21.8-3.0.

\section{PROPERTIES OF G21.8-3.0}

\subsection{Radio structure, polarisation and spectral index}

We show total-intensity $(I)$ images of G21.8-3.0 from the Urumqi $\lambda 6 \mathrm{~cm}$ survey, the new Effelsberg $\lambda 11 \mathrm{~cm}$ observations (a combination of the four individual bands) and the archived Effelsberg $\lambda 21 \mathrm{~cm}$ survey in the top panels of Fig. 1 . With the higher angular resolution of 4.8 , more details of G21.8-3.0 are visible at $\lambda 11 \mathrm{~cm}$. G21.8-3.0 has a nearly circular shape with a diameter of about $1^{\circ}$. Enhanced total-intensity emission is seen in a partial shell structure extending from northeast to north and a small circular section in the southeast. Three point-like sources were identified: G21.93-2.72 with a spectral index of $\alpha=-0.63$ (Vollmer et al. 2010), the planetary nebula G22.08-3.19 (Frew et al. 2013), and G21.66-2.79 about which not much information is available in literature. We list their flux densities at $\lambda 21 \mathrm{~cm}, \lambda 11 \mathrm{~cm}$, and $\lambda 6 \mathrm{~cm}$ in Table 1 . All $S_{\lambda 21 \mathrm{~cm}}$ values are retrieved from the NVSS source catalogue (Condon et al. 1998). The $S_{\lambda 11 \mathrm{~cm}}$ values of G21.93-2.72 and G21.66-2.79 are derived from Gaussian fits to the newly observed Effelsberg $\lambda 11 \mathrm{~cm}$ data and the $S_{\lambda 6} \mathrm{~cm}$ value for

2 http://astro.phys.wvu.edu/wise/ 



Figure 1. Radio continuum images of G21.8-3.0. Top panels from left to right: total intensity $I$ of G21.8-3.0 from the Urumqi $\lambda 6 \mathrm{~cm}$, Effelsberg $\lambda 11 \mathrm{~cm}$, and $\lambda 21 \mathrm{~cm}$ observations. The $\lambda 6 \mathrm{~cm}$ and $\lambda 21 \mathrm{~cm}$ data were extracted from the legacy Galactic plane surveys, while the $\lambda 11 \mathrm{~cm}$ data were newly obtained as described in Sect. 2.2 . The contours run at $3.0+(\mathrm{n}-1) \times 3.0 \mathrm{mK} T_{\mathrm{B}}$, at $15.0+(\mathrm{n}-1) \times 15.0 \mathrm{mK}$ $T_{\mathrm{B}}$, and $90+(\mathrm{n}-1) \times 60 \mathrm{mK} T_{\mathrm{B}}(\mathrm{n}=1,2,3 \ldots)$ in the $\lambda 6 \mathrm{~cm}, \lambda 11 \mathrm{~cm}$, and $\lambda 21 \mathrm{~cm}$ images, respectively. The two dashed rectangles in the $\lambda 11 \mathrm{~cm}$ image mark the regions for TT-plots. Middle panels from left to right: polarised intensity PI of G21.8-3.0 at $\lambda 6 \mathrm{~cm}$ and $\lambda 11$ $\mathrm{cm}$. The contours are the same as in the top panels. Bottom panels from left to right: SHASSA H $\alpha$ and IRIS $60 \mu \mathrm{m}$ images of G21.8-3.0 overlaid by $\lambda 11 \mathrm{~cm}$ contours.
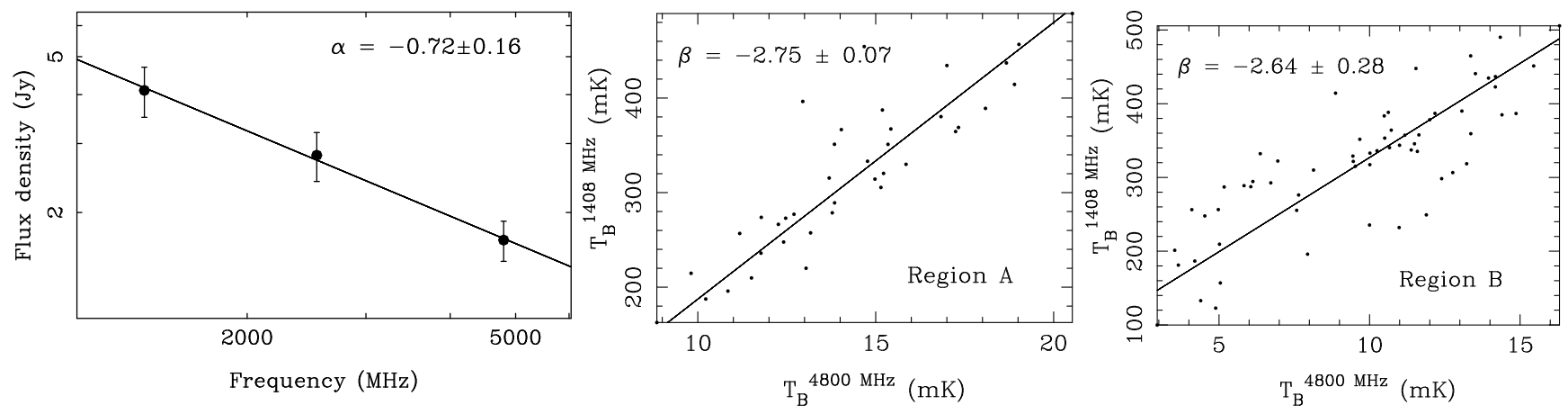

Figure 2. Left panel: radio continuum spectrum of G21.8-3.0 fitted by the flux densities obtained from the Urumqi $\lambda 6 \mathrm{~cm}$ data, the Effelsberg $\lambda 11 \mathrm{~cm}$ and $\lambda 21 \mathrm{~cm}$ data after removing the contributions from three point-like sources listed in Table 1. Middle and right panels: TT-plots by using the Urumqi $\lambda 6 \mathrm{~cm}$ data against Effelsberg $\lambda 21 \mathrm{~cm}$ data for the Region A (middle panel) and B (right panel) as indicated in the top middle panel of Fig. 1. 
G21.93-2.72 is taken from the PWN survey (Wright et al. 1994). Their contributions were subtracted so that the flux densities of the extended source G21.8-3.0 at these three bands can be estimated.

The $P I$ (Polarised Intensity, $P I=\sqrt{Q^{2}+U^{2}}$ ) images at $\lambda 6 \mathrm{~cm}$ and $\lambda 11 \mathrm{~cm}$ are shown in the middle panels of Fig. 1. The orientation of $B$-field vectors of the polarised emission seems to be uniform, implying the presence of regular magnetic fields. Strong polarised emission is seen outside G21.8-3.0 at $\lambda 6 \mathrm{~cm}$ without any corresponding totalintensity counterparts. These patches are either Faraday screens or caused by the turbulent magnetic fields as discussed in Sun et al. (2011). By comparing the polarisation angles $\left(P A \mathrm{~s}, P A=\frac{1}{2} \operatorname{atan} \frac{U}{Q}\right)$ at these two bands, the rotation measure $\left(R M, R M=\Delta P A /\left(\lambda_{6 \mathrm{~cm}}{ }^{2}-\lambda_{11 \mathrm{~cm}}{ }^{2}\right)\right)$ of the source can be estimated. For the region with prominent polarised emission, i.e. the eastern part of G21.8-3.0, we obtained an averaged $R M$ of $\sim 80 \mathrm{rad} \mathrm{m}^{-2}$ with $\mathrm{n} \pi$ ambiguity of $312 \mathrm{rad} \mathrm{m}^{-2}$ from the $\lambda 6 \mathrm{~cm}$ data and the combined $\lambda 11$ $\mathrm{cm}$ data (the central frequency was taken as $2536.25 \mathrm{MHz}$ ) convolved to 9'.5. This result was tested by the data at the high end $(2696.25 \mathrm{MHz})$ and the low end $(2376.25 \mathrm{MHz})$ of the new $\lambda 11 \mathrm{~cm}$ observations at the angular resolution of 4. 8 . We got a consistent $R M$ of $\sim 90 \mathrm{rad} \mathrm{m}^{-2}$. The $\mathrm{n} \pi$ ambiguity is about $\pm 864 \mathrm{rad} \mathrm{m}^{-2}$, which seems very unlikely and implies that $R M \sim 80-90 \mathrm{rad} \mathrm{m}^{-2}$ of G21.8-3.0 is most likely convincing. No $R M \mathrm{~s}$ of extra-Galactic sources behind G21.8-3.0 are available to diminish the ambiguity (Xu \& Han 2014).

The radio continuum spectral index is a key to distinguish the non-thermal synchrotron emission from SNRs and thermal bremsstrahlung radiation from H II regions. Most shell-type SNRs have a spectral index around $\alpha \sim-0.5$ $\left(S_{\nu} \sim \nu^{\alpha}\right)$ (see Urošević 2014; Dubner 2017), which makes them distinct from the optically-thin emission of $\mathrm{H}$ II regions with a much flatter spectrum of $\alpha \sim-0.1$ (see Wilson et al. 2013). To estimate the flux densities of G21.8-3.0 at each frequency, a twisted plane was fitted and subtracted in each map to remove the unrelated foreground/background emission before integration. We got $S_{\lambda 6 \mathrm{~cm}}=1.7 \pm 0.2 \mathrm{Jy}, S_{\lambda 11 \mathrm{~cm}}$ $=2.8 \pm 0.4 \mathrm{Jy}$, and $S_{\lambda 21 \mathrm{~cm}}=4.1 \pm 0.6 \mathrm{Jy}$ for G21.8-3.0 after the point-like sources were subtracted. The radio continuum spectrum was fitted and is shown in the left panel of Fig. 2. The spectral index is $\alpha=-0.72 \pm 0.16$, which clearly indicates the non-thermal nature of G21.8-3.0.

The TT (Temperature - Temperature) plot method introduced by Turtle et al. (1962) has been widely used to reveal the spectral index of an extended emission because it is insensitive to zero-level uncertainties of images. Taking the notion that $T_{\text {source }}(\nu) \sim \nu^{\beta}$, where $T_{\text {source }}$ is the temperature of the target source and $\beta$ is defined as the brightness temperature spectral index, the temperature map observed at frequency $\nu$ can be described as $T(\nu)=T_{\text {source }}(\nu)+C$. Here, $C$ is the baselevel offset. By substituting $T_{\text {source, }}$, it is then straightforward to have $T\left(\nu_{1}\right)=\left(\frac{\nu_{1}}{\nu_{2}}\right)^{\beta} T\left(\nu_{2}\right)-\left(\frac{\nu_{1}}{\nu_{2}}\right)^{\beta} C_{2}+C_{1}$, implying that the slope of linear regression in TT-plots can be used to derive source spectral index $\beta$ and the baselevel offsets included in the intercept do not have any influence on the slope. Considering the flux density $S \sim 2 k T \nu^{2} \sim \nu^{\alpha}$ for the radio image and $T \sim \nu^{\beta}$, one can relate the flux density and brightness temperature spectral indices as $\alpha=\beta+2$. For G21.8-3.0, two areas were selected (marked as A and B in the top middle panel of Fig. 1) for TT-plots, which are free from point-like source contamination by inspecting the high angular resolution NVSS image. Region A is the shell-like structure in the northeastern part of G21.8-3.0, and Region $\mathrm{B}$ is an area which is relatively weak in emission. The TT-plots give the brightness temperature spectral indices as being $\beta=-2.75 \pm 0.07$ and $\beta=-2.64 \pm 0.28$ between the $\lambda 6$ $\mathrm{cm}$ and $\lambda 21 \mathrm{~cm}$ data for Region $\mathrm{A}$ and $\mathrm{B}$, respectively, very consistent with the result derived from the flux densities at the three bands.

Based on the flux density measured at $\lambda 6 \mathrm{~cm}$ and the fitted spectral index of $\alpha=-0.72$, we estimated the surface brightness of G21.8-3.0 at $1 \mathrm{GHz}$ to be $\Sigma_{1 \mathrm{GHz}}=2.2 \pm 0.3 \times$ $10^{-22}\left[\mathrm{Wm}^{-2} \mathrm{~Hz}^{-1} \mathrm{sr}^{-1}\right]$, placing it at the fainter end of the known Galactic SNRs (see Fig. 1 in Green 2014).

\subsection{Properties at optical, infrared and high-energy bands}

Some SNRs do show features at optical and infrared bands, and a few were even newly identified from optical observations, e.g. SNRs G159.6+7.3 and G70.0-21.5 discovered through deep H $\alpha$ observations (Fesen \& Milisavljevic 2010; Fesen et al. 2015).

We extracted the continuum-corrected $\mathrm{H} \alpha$ emission from SHASSA (Gaustad et al. 2001) for the G21.8-3.0 field as shown in the bottom left panel of Fig. 1. The image was slightly smoothed to $4^{\prime}$ from the original 3'.2 resolution. For the planetary nebula G22.08-3.19, bright $\mathrm{H} \alpha$ emission is visible. Some diffuse $\mathrm{H} \alpha$ emission overlaps the lower part of G21.8-3.0, however, no solid correlation can be established. The most interesting feature seen in the $\mathrm{H} \alpha$ image is an elongated filament nicely running along the northern edge of G21.8-3.0. It is necessary to confirm with other optical lines, such as [S II] and [O I] (e.g. Fesen et al. 1985; Kopsacheili et al. 2019) whether it is a shock emission filament.

The IRIS $60 \mu \mathrm{m}$ image of G21.8-3.0 is shown in the bottom middle panel of Fig. 1. The WISE images, which we did not present, resemble the structures in the IRIS map, but show more point sources due to their higher angular resolution. No catalogued WISE H II regions (Anderson et al. 2014) are found in this region. SNRs have faint far-infrared emission compared to H II regions (Fürst et al. 1987) and lack mid-infrared emission (Anderson et al. 2017). No clear morphological correlation is found between the infrared (IRIS and WISE) and radio images of G21.8-3.0. Based on the comparison between the $60 \mu \mathrm{m}$ infrared and Effelsberg $\lambda 11 \mathrm{~cm}$ data, Fürst et al. (1987) found that the infrared to radio continuum ratio $R$ is as high as $1000 \pm 500$ for H II regions, and proposed that the $\lambda 11 \mathrm{~cm}$ sources with $R<$ 250 could be SNR candidates. We found low $R$ values, i.e. $R \sim 80$ in the lower part and $R \sim 240$ in the upper part of G21.8-3.0, which support its SNR nature.

No X-ray counterpart was found for G21.8-3.0 from the ROSAT X-ray All-Sky Survey data in both low (0.1 $0.4 \mathrm{keV})$ and high $(0.4-2.4 \mathrm{keV})$ bands (Voges et al. 1999) and the archived data from the Chandra X-ray observatory. We also failed to find any discrete source that might be associated with G21.8-3.0 in the high-energy Galactic plane surveys from HESS and HAWC (Jardin-Blicq et al. 2019) and 

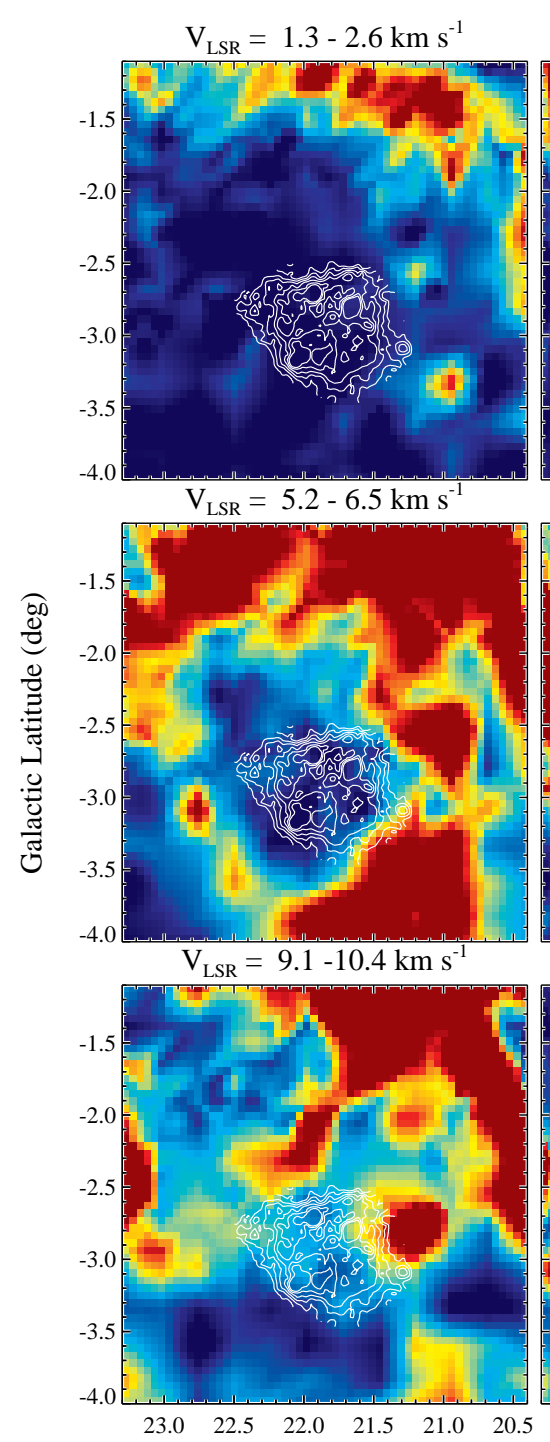

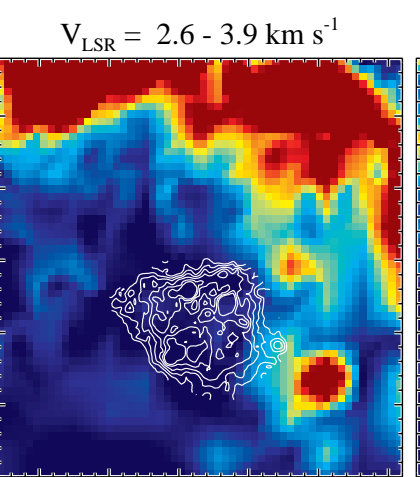

$\mathrm{V}_{\mathrm{LSR}}=6.5-7.8 \mathrm{~km} \mathrm{~s}^{-1}$

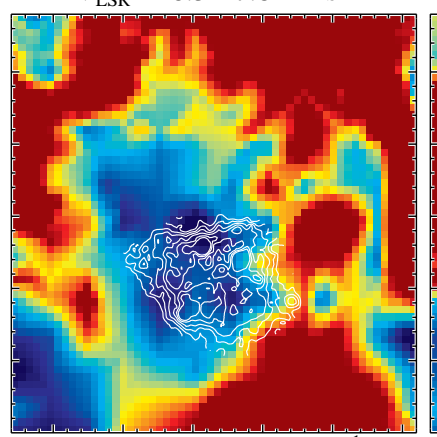

$\mathrm{V}_{\mathrm{LSR}}=10.4-11.7 \mathrm{~km} \mathrm{~s}^{-1}$

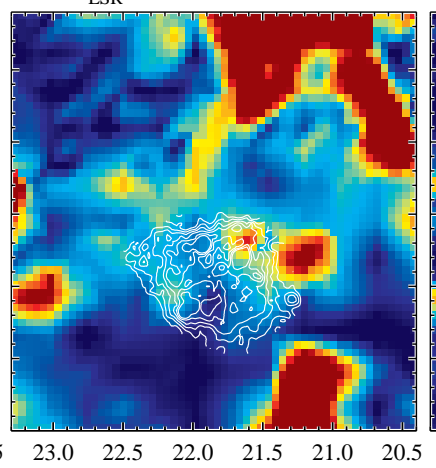

$\mathrm{V}_{\mathrm{LSR}}=3.9-5.2 \mathrm{~km} \mathrm{~s}^{-1}$
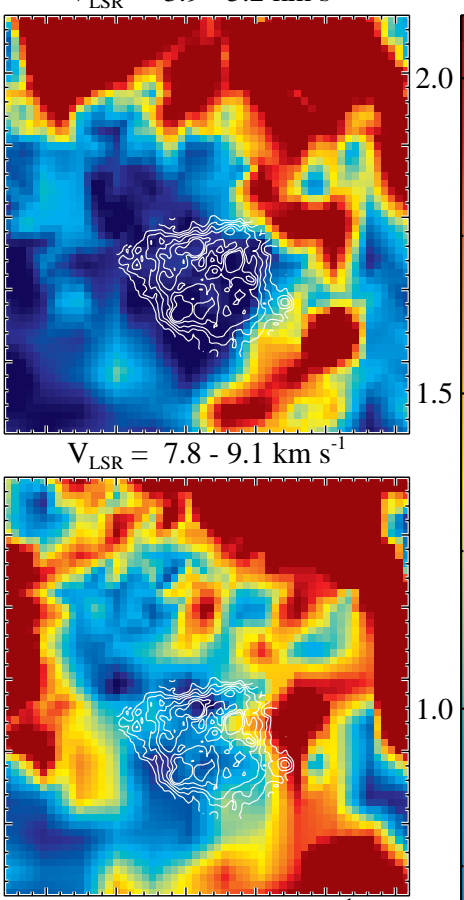

$\mathrm{V}_{\mathrm{LSR}}=11.7-13.0 \mathrm{~km} \mathrm{~s}^{-1}$

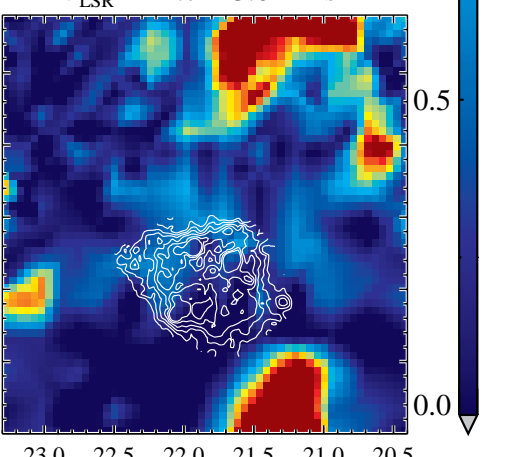

Galactic Longitude (deg)

Figure 3. Intensity maps of ${ }^{12} \mathrm{CO}(J=1-0)$ in the area of $\mathrm{G} 21.8-3.0$ showing integrated emission in $1.3 \mathrm{~km} \mathrm{~s}^{-1}$ wide channels for the velocity range of $1.3-13.0 \mathrm{~km} \mathrm{~s}^{-1}$, overlaid by contours of total intensity $I$ of Effelsberg $\lambda 11 \mathrm{~cm}$ observations.

the Fermi 4FGL catalogue (The Fermi-LAT collaboration 2019).

\subsection{Possibly associated gas structure and the distance}

Among the 294 known Galactic SNRs (Green 2019), 70 of them have physical contact with the surrounding molecular clouds (MCs, e.g. Chen et al. 2014). The kinematic distance therefore can be estimated from the systematic velocity of the associated MCs (e.g. Foster et al. 2013). We explored the possible SNR-MC association i.e. morphological agreement or corresponding MC features (e.g. cavities, bubbles) with that of G21.8-3.0.

The ${ }^{12} \mathrm{CO}(J=1-0)$ survey data from Dame et al. (2001), which have an angular resolution of $\sim 8 ! 4$ and a velocity resolution of $0.65 \mathrm{~km} \mathrm{~s}^{-1}$ (see Table 1 of Dame et al. 2001), are adopted in this work. As shown in Fig. 4, we aver- aged the CO spectra in the sky area surrounding G21.8-3.0 (see Fig. 3) and found two major emission features near $V_{\mathrm{LSR}} \sim 8 \mathrm{~km} \mathrm{~s}^{-1}$ and $\sim 55 \mathrm{~km} \mathrm{~s}^{-1}$, respectively. By checking the channel maps around $V_{\mathrm{LSR}} \sim 55 \mathrm{~km} \mathrm{~s}^{-1}$, the emission is very extended and originates from the Galactic plane, i.e. $b>-1.8$, which is 0.7 above the radio continuum emission, hence is not related to G21.8-3.0. For the emission component of $V_{\mathrm{LSR}} \sim 8 \mathrm{~km} \mathrm{~s}^{-1}$, we show the ${ }^{12} \mathrm{CO}(J=1-0)$ intensity maps integrated in the velocity range of $1.3-13.0 \mathrm{~km} \mathrm{~s}^{-1}$ in steps of $1.3 \mathrm{~km} \mathrm{~s}^{-1}$ in Fig. 3. A cavity-like CO feature is seen to encircle the radio-continuum emission of G21.8-3.0 in the velocity range of $V_{\mathrm{LSR}}=3.9-7.8 \mathrm{~km} \mathrm{~s}^{-1}$. The southwestern edge of G21.8-3.0 follows the wall of the cavity. The northeastern part of the cavity extends beyond the continuum emission, and is not coincident with any discrete sources in the Urumqi $\lambda 6 \mathrm{~cm}$ image. In the velocity channels of $V_{\mathrm{LSR}}<3.9 \mathrm{~km} \mathrm{~s}^{-1}$ or $>7.8 \mathrm{~km} \mathrm{~s}^{-1}$, the cavity becomes weak and disappears. 


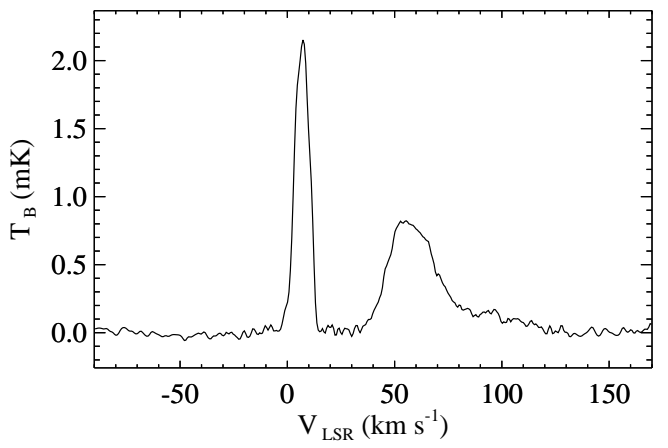

Figure 4. Averaged spectrum of ${ }^{12} \mathrm{CO}(J=1-0)$ for the area shown in Fig. 3.

The systematic velocity of this cavity-like $\mathrm{CO}$ emission peaks at $V_{\mathrm{LSR}}=5.9 \pm 2 \mathrm{~km} \mathrm{~s}^{-1}$, which is used to estimate the kinematic distance of G21.8-3.0 by adopting Galaxy rotation models.

Two methods were used. One is the revised kinematic distance calculator provided by Reid et al. (2014) with the parameters of $R_{0}=8.3 \mathrm{kpc}$ (distance from the Sun to the Galactic centre), $\Theta_{0}=240 \mathrm{~km} \mathrm{~s}^{-1}$ (circular rotation of the local standard of rest), and the revised Solar motions. The derived nearer kinematic distance is $0.42_{-0.15}^{+0.61} \mathrm{kpc}$. The other method is a parallax-based distance estimator for Galactic sources (Reid et al. 2016): the estimated distance is $0.25 \pm 0.13 \mathrm{kpc}$, comparable to that of the nearest Galactic SNR Vela (Caraveo et al. 2001). These two results are consistent within their errors, locating G21.8-3.0 in the Local Arm (e.g. Xu et al. 2018). Based on Green (2019), there are less than 10 SNRs that have a measured distance within $1 \mathrm{kpc}$. The discovery of G21.8-3.0 would enhance the nearEarth SNR rate and it probably has made some impact on Earth as discussed in Firestone (2014).

In comparison with the northeastern shell of G21.8-3.0, the radio emission of the southwestern edge, visually contacting the CO clouds, is relatively weak and does not form a shell, which might imply that the progenitor star exploded in a pre-existing molecular cavity and the free-expansion shock just reached or interacted with the wall.

We checked the H I 21-cm survey data of the Parkes Galactic all-sky survey (McClure-Griffiths et al. 2009; Kalberla et al. 2010), which has an effective angular resolution of $\sim 16^{\prime}$ with a velocity resolution of $1.0 \mathrm{~km} \mathrm{~s}^{-1}$. The H I emission in this region is very diffuse and complex. No related structures can be firmly identified in the velocity range of $-50 \mathrm{~km} \mathrm{~s}^{-1}-200 \mathrm{~km} \mathrm{~s}^{-1}$.

\subsection{Size, age and brightness}

Taking the average kinematic distance of 330 pc derived from the CO data, the diameter of G21.8-3.0 is just about 6 pc. Such a small physical size is an indication of young SNRs. The spectral index of G21.8-3.0, $\alpha=-0.72$, resem- bles that of some historical SNRs such as Cas A ( $\alpha=-0.77)$, SN $1006(\alpha=-0.6)$ and Tycho $(\alpha=-0.58)$ (Green 2019). However, unlike the radial magnetic fields present in these young SNRs (Reynoso et al. 2013; Reich 2016), the current images for polarised emission of G21.8-3.0 seems to be patchy (see Fig. 1). Multi-frequency polarisation observations with better angular resolution are needed to clarify the detailed configuration of the magnetic fields in G21.8-3.0.

In the case that G21.8-3.0 is not young, the small physical size requires a low initial explosion energy and/or a high ambient density into which it expands (Sedov 1959). However, as we failed to identify any related H I structures, it is difficult to estimate the initial ambient number density and further the age of G21.8-3.0 as done by Su et al. (2017) and Kothes et al. (2017). For the shell-type SNRs, distance and diameter can also be estimated from the empirical radio surface brightness - diameter $(\Sigma-\mathrm{D})$ relation. By collecting 65 shell-type SNRs with direct distance estimates as the calibration sample, Pavlović et al. (2014) found a new steeper slope for the $\Sigma$-D relation. Based on this relation, we get a physical size of more than $40 \mathrm{pc}$ for the case of G21.8-3.0, so that the distance of this SNR is larger than $2.3 \mathrm{kpc}$, much more distant than the value obtained from the CO cavity. Note that distances estimated from the $\Sigma$-D relation have an uncertainty as large as $35 \%$ or more due to several facts, e.g. the intrinsic properties of SNRs, the environments where SNRs evolve, and the selection effects of the calibration sample (Green 1984; Pavlović et al. 2013; Kostić et al. 2016). We therefore emphasise that the distance of G21.8-3.0 is still an open issue, which may be settled by new $\mathrm{CO} / \mathrm{HI}$ data with higher angular resolution and sensitivity or future finding of $1720-\mathrm{MHz} \mathrm{OH}$ masers due to SNR-MC interactions, or even using red clump stars or dust extinction (e.g. Shan et al. 2018; Yu et al. 2019).

\subsection{Associated pulsar?}

We searched in the ATNF pulsar catalogue ${ }^{4}$ for associated pulsars, that may also hint the distance of G21.8-3.0. PSR J1843-1113 $\left(\ell=22.06, b=-3^{\circ} .40\right)$ with a distance of $1.26 \mathrm{kpc}$ is outside G21.8-3.0 but not far away. In the case that it is a run-away pulsar from the centre, the proper motion should confirm this. We found that the measured velocity vector of PSR J1843-1113 (Desvignes et al. 2016) did not match the presumed path, i.e. tracing back to the centre of G21.8-3.0, therefore, seems not to be related. PSR $\mathrm{J} 1840-1122\left(\ell=21^{\circ} .56, b=-2^{\circ} .74\right.$, Hobbs et al. 2004) overlaps G21.8-3.0. The distance of the pulsar is about $8.21 \mathrm{kpc}$, on the Far 3-kpc Arm according to Hou \& Han (2014). No proper motion information is available yet. If this SNRpulsar association could be confirmed, the physical size of G21.8-3.0 would be 140 pc, which is exceptionally large. This is unlikely and the overlap may be just a coincidence.

No pulsars were identified as stated above implying the progenitor of G21.8-3.0 might not be a massive star. No flatspectrum component was seen inside G21.8-3.0, indicating the non-existence of a pulsar wind nebula at the current detection sensitivity.

${ }^{4}$ http://www.atnf.csiro.au/research/pulsar/psrcat/ 


\section{SUMMARY}

Sensitive large-scale radio continuum surveys are crucial for discovering Galactic SNRs with low surface brightness. By identifying radio sources in the Sino-German $\lambda 6 \mathrm{~cm}$ polarisation survey of the Galactic plane, we serendipitously discovered the extended object G21.8-3.0, which has a circular shape with a diameter of about $1^{\circ}$. To reveal the radio emission properties, new $\lambda 11 \mathrm{~cm}$ observations were carried out with the Effelsberg 100-m radio telescope. Together with the Effelsberg $\lambda 21 \mathrm{~cm}$ data, the radio continuum spectral index was derived to be $\alpha_{6 \mathrm{~cm}-21 \mathrm{~cm}}=-0.72 \pm 0.16$, consistent with that of synchrotron emission from SNRs. Polarised emission from G21.8-3.0 was detected both at $\lambda 6 \mathrm{~cm}$ and $\lambda 11 \mathrm{~cm}$. By comparing polarisation angles, the $R M$ of G21.8-3.0 was estimated to be $\sim 80-90 \mathrm{rad} \mathrm{m}^{-2}$. An $\mathrm{H} \alpha$ filament is seen to run along the northern edge of G21.8-3.0. The weak infrared emission of the source exemplifies its SNR nature. A cavity-like $\mathrm{CO}$ feature was found in the direction of G21.8-3.0 at $V_{\mathrm{LSR}}=5.9 \pm 2 \mathrm{~km} \mathrm{~s}^{-1}$. If related to G21.8-3.0, its distance is about $330 \mathrm{pc}$, which means it is a local-arm object, however, the $\Sigma$-D relation for shell-type SNRs results in a much farther distance.

\section{ACKNOWLEDGEMENTS}

We thank the anonymous referee for useful comments that improved this work, and Dr. James Wicker and Dr. David Champion for their kindness on language editing. We would like to thank Dr. Alex Kraus and Dr. Peter Müller for support with the observations and data reduction, and also the electronics group at Effelsberg for connecting the SPECPOL polarimeter with the $\lambda 11 \mathrm{~cm}$ receiver. This research is based in part on observations with the Effelsberg 100-m telescope of the MPIfR, Bonn. The Chinese authors are supported by the National Natural Science Foundation of China (No. 11988101, U1831103, 11933011, 11833009), the National Key R\&D Program of China (No. 2017YFA0402701), the Open Project Program of the Key Laboratory of FAST, NAOC, the Chinese Academy of Sciences, and the Partner group of the MPIfR at NAOC in the framework of the exchange programme between MPG and CAS for many bilateral visits. XYG acknowledges financial support from the CAS-NWO cooperation programme. LGH is grateful for the support by the Youth Innovation Promotion Association CAS.

\section{REFERENCES}

Anderson L. D., Bania T. M., Balser D. S., Cunningham V., Wenger T. V., Johnstone B. M., Armentrout W. P., 2014, ApJS, 212, 1

Anderson L. D., et al., 2017, A\&A, 605, A58

Brogan C. L., Gelfand J. D., Gaensler B. M., Kassim N. E., Lazio T. J. W., 2006, ApJ, 639, L25

Caraveo P. A., De Luca A., Mignani R. P., Bignami G. F., 2001, ApJ, 561, 930

Chen Y., Jiang B., Zhou P., Su Y., Zhou X., Li H., Zhang X., 2014, in Ray A., McCray R. A., eds, IAU Symposium Vol. 296, Supernova Environmental Impacts. pp 170-177 (arXiv: 1304.5367), doi:10.1017/S1743921313009423

Condon J. J., Cotton W. D., Greisen E. W., Yin Q. F., Perley R. A., Taylor G. B., Broderick J. J., 1998, AJ, 115, 1693
Dame T. M., Hartmann D., Thaddeus P., 2001, ApJ, 547, 792

Desvignes G., et al., 2016, MNRAS, 458, 3341

Dubner G., 2017, Radio Emission from Supernova Remnants, Handbook of Supernovae Springer International Publishing AG, 2017. p. 2041, doi:10.1007/978-3-319-21846-5_91

Ferrand G., Safi-Harb S., 2012, Advances in Space Research, 49, 1313

Fesen R. A., \& Milisavljevic D., 2010, AJ, 140, 1163

Fesen R. A., Blair W. P., Kirshner R. P., 1985, ApJ, 292, 29

Fesen R. A., Neustadt J. M. M., Black C. S., Koeppel A. H. D., 2015, ApJ, 812, 37

Firestone R. B., 2014, ApJ, 789, 29

Foster T. J., Cooper B., Reich W., Kothes R., West J., 2013, A\&A, 549, A107

Frew D. J., Bojičić I. S., Parker Q. A., 2013, MNRAS, 431, 2

Fürst E., Reich W., Sofue Y., 1987, A\&AS, 71, 63

Gao X. Y., \& Han J. L., 2014, A\&A, 567, A59

Gao X. Y., et al., 2010, A\&A, 515, A64

Gao X. Y., Sun X. H., Han J. L., Reich W., Reich P., Wielebinski R., 2011, A\&A, 532, A144

Gaustad J. E., Rosing W., McCullough P., Van Buren D., 2001, in B. Paczynski, W.-P. Chen, \& C. Lemme ed., Astronomical Society of the Pacific Conference Series Vol. 246, IAU Colloq. 183: Small Telescope Astronomy on Global Scales. p. 75

Gerbrandt S., Foster T. J., Kothes R., Geisbüsch J., Tung A., 2014, A\&A, 566, A76

Green D. A., 1984, MNRAS, 209, 449

Green D. A., 2014, in Ray A., McCray R. A., eds, IAU Symposium Vol. 296, Supernova Environmental Impacts. pp 188-196 (arXiv: 1309.3072), doi:10.1017/S1743921313009459

Green D. A., 2019, Journal of Astrophysics and Astronomy, 40,36

Hobbs G., Lyne A. G., Kramer M., Martin C. E., Jordan C., 2004, MNRAS, 353, 1311

Hou L. G., \& Han J. L., 2014, A\&A, 569, A125

Jardin-Blicq A., Marandon V., Brun F., 2019, in 36th International Cosmic Ray Conference (ICRC2019). p. 706 (arXiv:1908.06658)

Kalberla P. M. W., et al., 2010, A\&A, 521, A17

Kopsacheili M., Zezas A., Leonidaki I., 2019, arXiv e-prints, p. arXiv:1909.05879

Kostić P., Vukotić B., Urošević D., Arbutina B., Prodanović T., 2016, MNRAS, 461, 1421

Kothes R., Sun X. H., Reich W., Foster T. J., 2014, ApJ, 784, L26

Kothes R., Reich P., Foster T. J., Reich W., 2017, A\&A, 597, A116

Landecker T. L., et al., 2010, A\&A, 520, A80

Li Z., Wheeler J. C., Bash F. N., Jefferys W. H., 1991, ApJ, 378, 93

McClure-Griffiths N. M., et al., 2009, ApJS, 181, 398

Miville-Deschênes M., \& Lagache G., 2005, ApJS, 157, 302

Padmanabhan T., 2001, Theoretical Astrophysics - Volume 2, Stars and Stellar Systems. Cambridge University Press, doi:10.2277/0521562414

Pavlović M. Z., Urošević D., Vukotić B., Arbutina B., Göker Ü. D., 2013, ApJS, 204, 4

Pavlović M. Z., Dobardzic A., Vukotic B., Urosevic D., 2014, Serbian Astronomical Journal, 189, 25

Reich W., 2016, in Supernova Remnants: An Odyssey in Space after Stellar Death. p. 60

Reich W., Reich P., Fürst E., 1990, A\&AS, 83, 539

Reid M. J., et al., 2014, ApJ, 783, 130

Reid M. J., Dame T. M., Menten K. M., Brunthaler A., 2016, ApJ, 823, 77

Reynoso E. M., Hughes J. P., Moffett D. A., 2013, AJ, 145, 104

Sedov L. I., 1959, Similarity and Dimensional Methods in Mechanics, Academic Press, New York 
Shan S. S., Zhu H., Tian W. W., Zhang M. F., Zhang H. Y., Wu

D., Yang A. Y., 2018, ApJS, 238, 35

Stil J. M., et al., 2006, AJ, 132, 1158

Su Y., Zhou X., Yang J., Chen Y., Chen X., Gong Y., Zhang S., 2017, ApJ, 845, 48

Sun X. H., Reich W., Han J. L., Reich P., Wielebinski R., 2006, A\&A, 447, 937

Sun X. H., Han J. L., Reich W., Reich P., Shi W. B., Wielebinski R., Fürst E., 2007, A\&A, 463, 993

Sun X. H., Reich W., Han J. L., Reich P., Wielebinski R., Wang C., Müller P., 2011, A\&A, 527, A74

Tammann G. A., Loeffler W., Schroeder A., 1994, ApJS, 92, 487

The Fermi-LAT collaboration 2019, arXiv e-prints, p. arXiv:1902.10045

Turtle A. J., Pugh J. F., Kenderdine S., Pauliny-Toth I. I. K., 1962, MNRAS, 124, 297

Urošević D., 2014, Ap\&SS, 354, 541

Voges W., et al., 1999, A\&A, 349, 389

Vollmer B., et al., 2010, A\&A, 511, A53

Wilson T. L., Rohlfs K., Hüttemeister S., 2013, Tools of Radio Astronomy, 6th edn. Springe-Verlag, Berlin, doi:10.1007/978-3-642-39950-3.

Wright A. E., Griffith M. R., Burke B. F., Ekers R. D., 1994, ApJS, 91, 111

Wright E. L., et al., 2010, AJ, 140, 1868

Xiao L., Han J. L., Reich W., Sun X. H., Wielebinski R., Reich P., Shi H., Lochner O., 2011, A\&A, 529, A15

Xu J., \& Han J. L., 2014, MNRAS, 442, 3329

Xu Y., Hou L.-G., Wu Y.-W., 2018, Research in Astronomy and Astrophysics, 18, 146

Yu B., Chen B. Q., Jiang B. W., Zijlstra A., 2019, MNRAS, 488,3129

This paper has been typeset from a $\mathrm{T}_{\mathrm{E}} \mathrm{X} / \mathrm{LAT}_{\mathrm{E}} \mathrm{X}$ file prepared by the author. 